\title{
Local-global processing in the upper versus lower visual fields
}

\author{
STEPHEN D. CHRISTMAN \\ University of Toledo, Toledo, Ohio
}

\begin{abstract}
Two experiments examining differences in the processing of local versus global information as a function of visual field location are reported. Both experiments demonstrated the presence of upper versus lower visual field advantages in the processing of local versus global information, respectively. In addition, previous findings of right versus left visual field advantages in the processing of local versus global information, respectively, were partially replicated. Results are interpreted in terms of Previc's (1990) hypothesis of functional specialization of the visual field.
\end{abstract}

In a recent review article, Previc (1990) proposed that the upper versus lower visual fields in humans are functionally specialized for the processing of local versus global information, respectively. Previc offers intriguing ecological arguments about how the roles of the upper versus lower visual fields in far (extrapersonal space) versus near (peripersonal space) vision led to their respective specializations. Specifically, Previc argues that the lower visual field has become specialized for near vision, because objects located nearer to an observer tend to be located lower in the visual field. In turn, because the processing of objects in near space typically involves visuomotor manipulation (i.e., reaching and grasping), the lower visual field has become specialized for global processing. The need for global processing in near vision arises, for example, from the fact that the image of one's hand while reaching for an object is both blurred (due to its motion) and diplopic (due to the typical pattern of fixation on the more distal object that is being reached for). Previc suggests, therefore, that channels carrying lower visual field information became specialized for "global perception" in order to adequately deal with the optically degraded visual input. Conversely, Previc argues that the upper visual field has come to play a predominant role in far vision, because more distal objects are typically located higher in the field of vision due to the vertical constraints of gravity. In turn, because the processing of objects in far space typically involves visual search and the processing of finer details of form, Previc suggests that the upper visual field has become specialized for local processing.

Besides the potential significance of Previc's (1990) hypothesis for general theories of vision, his ideas also bear

An earlier version of this paper was presented at the 32nd Annual Meeting of the Psychonomic Society, San Francisco, CA, November 1991. Preparation of this article was supported in part by a Faculty Research and Fellowship Award from the University of Toledo Office of Research. I wish to thank Frederick Kitterle for helpful comments and Susan Mooney for assistance in data collection. Correspondence should be addressed to S. Christman, Department of Psychology, University of Toledo, Toledo, $\mathrm{OH} 43606$. an important relation to the interpretation of left versus right visual field differences. As Bryden and Underwood (1990) point out in their commentary on Previc's article, his proposal bears a similarity to neuropsychological evidence for functional specialization of the right versus left visual fields for the processing of local versus global information, respectively (e.g., Robertson \& Lamb, 1991; Sergent, 1982).

Although Previc reviews and attempts to synthesize a considerable body of neurophysiological and behavioral evidence in support of the hypothesis regarding upper versus lower visual field differences, no direct empirical evidence is presented regarding the proposed local-global distinction. The purpose of the current paper, therefore, was to examine the processing of local versus global visual information presented along the vertical, horizontal, and oblique meridians of the visual field. As an example of local-global stimuli, Previc offers the hierarchical letter stimuli developed by Navon (1977), consisting of a large letter made up in outline from smaller letters. These are also the stimuli typically employed in studies investigating left-right visual field differences in local-global processing, and will be utilized in the current experiments.

\section{EXPERIMENT 1}

\section{Method}

Subjects. Sixteen subjects with normal or corrected-to-normal vision participated for course credit. All were right-handed with no left-handed relatives in their immediate family (as assessed by a brief questionnaire), and all were naive about the purpose of the study.

Apparatus and Stimuli. Stimulus presentations were controlled by MacLaboratory Psychology software (V. 2.0) running on a Macintosh II computer. Stimuli were displayed on an AppleColor high-resolution RGB monitor. At a viewing distance of $70 \mathrm{~cm}$, the active video display area subtended $14.1^{\circ} \times 18.6^{\circ}$ of visual angle. All stimulus events were black on a white surround. Display luminance was $95 \mathrm{~cd} / \mathrm{m}^{2}$.

The stimuli consisted of the hierarchical letter stimuli composed of the letters $E$ and $H$. The large global letter was constructed from small letters (18-point Helvetica) arranged within a $5 \times 5$ matrix. The overall stimulus subtended $2.0^{\circ} \times 2.9^{\circ}$, and the inner edge of each stimulus was located $1.6^{\circ}$ from a small " $x$ " in the center of the screen, which served as a fixation point. Four locations were used: directly above, below, left of, and right of the fixation point. 
Procedure. A $250-\mathrm{msec}$ tone signaled the beginning of each trial, followed by a 500 -msec pause and then a 100 -msec stimulus presentation. The subjects' task was to determine whether or not a target letter had been presented. If the target letter occurred at either the global level, local level, or both levels, they were instructed to press a key on a keyboard in front of them with the index finger of one hand; if the target letter did not occur at either level, they pressed another key with the index finger of the other hand. Half the subjects responded to target present with their left hand; the other half responded to target present with their right hand. Similarly, half the subjects responded to the letter $\mathrm{E}$ as the target; the other half responded to the letter $\mathrm{H}$ as the target. Hand of response and target identity were counterbalanced. A 2-sec intertrial interval followed each response.

In each block of trials, there were equal numbers of target and nontarget trials. Each block consisted of 144 trials: 24 trials in which the target occurred only as the large letter $(\mathrm{L}+\mathrm{S}-), 24$ in which the target occurred only as the small letter $(\mathrm{L}-\mathrm{S}+), 24$ in which the target occurred as both letters $(\mathrm{L}+\mathrm{S}+)$, and 72 in which the target did not occur at either level $(\mathrm{L}-\mathrm{S}-)$. Each stimulus type occurred at each location an equal number of times. The subjects were run through three blocks of experimental trials for a total of 432 trials. They also received a block of 24 practice trials at the beginning of the session.

\section{Results and Discussion}

The average error rate across conditions was $2.6 \%$, so analyses were restricted to the reaction time (RT) data. A two-way analysis of variance (ANOVA) was performed, with stimulus type $(\mathrm{L}+\mathrm{S}+, \mathrm{L}+\mathrm{S}-, \mathrm{L}-\mathrm{S}+$, and $\mathrm{L}-\mathrm{S}-$ ) and stimulus location (upper, lower, left, and right visual fields) as within-subject variables. A main effect of stimulus type was obtained $[F(3,45)=15.48$, $p<.001$ ], with RT being shorter when the target letter appeared at both the local and global levels than when it appeared at only one or the other level. There was no main effect of location $[F(3,45)=1.33, p>.25]$. Finally, there was a significant interaction between stimulus type and location $[F(9,135)=5.10, p<.001]$.

In order to determine whether local-global processing differences exist as a function of visual hemifield, planned comparisons were conducted on the data from the trial types in which the target was present at only the global level (i.e., $\mathrm{L}+\mathrm{S}-$ ) versus only the local level (i.e., $\mathrm{L}-\mathrm{S}+$ ). These data are shown in Figure 1. In addition, analyses of effects of location were restricted to compar-

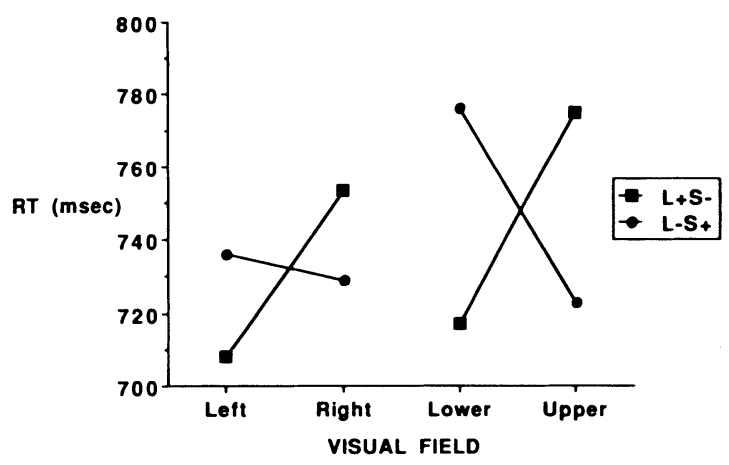

Figure 1. Reaction time as a function of stimulus type $(\mathrm{L}+\mathrm{S}-$ : global target only; $L-S+$ : local target only) and visual field location in Experiment 1. isons of left versus right visual field and upper versus lower visual field. For the $\mathrm{L}+\mathrm{S}-$ trials, there was a trend toward a left visual field advantage (VFA) $[F(1,15)=$ $4.13, p<.06]$ and a significant lower VFA $[F(1,15)=$ $15.89, p<.001]$. For the $\mathrm{L}-\mathrm{S}+$ trials, there was no difference between the left and right visual fields $(F<1)$ and a significant upper VFA $[F(1,15)=10.10, p<.01]$.

These results provide support for the hypothesis that the upper and lower visual fields are differentially specialized for the processing of local versus global information. When the target was present at the global level only, performance in the lower visual field was superior to that in the upper visual field; on the other hand, when the target was only present at the local level, performance was better in the upper visual field. Interestingly, these effects were more robust than the obtained left-right visual field effects, which failed to attain full significance for the global-only or local-only conditions. This finding does not necessarily call into question the validity of theories positing hemispheric differences in local-global processing, since Van Kleeck (1989) recently argued that although individual studies may fail to yield significant left-right visual field differences in local-global processing, the trends are virtually always in the hypothesized direction. Furthermore, in a meta-analysis, Van Kleeck found that the pooled results of extant studies clearly demonstrate left-right differences. However, the results of the current experiment demonstrate that the magnitude and/or reliability of upper-lower visual field differences in localglobal processing may exceed that of left-right differences.

\section{EXPERIMENT 2}

In Experiment 1, it was demonstrated that upper versus lower visual field differences occur in the processing of local and global information, and that the magnitude of these differences may surpass that of left versus right visual field differences. However, the fact that global precedence was not obtained with the stimuli from Experiment 1 limits the generalizability of the results, since faster processing of the global dimension is typically found. The purpose of Experiment 2, therefore, was to extend the paradigm from Experiment 1 to a situation in which global precedence is obtained. In addition, stimulus locations along the oblique meridians of the visual field were incorporated.

\footnotetext{
Method

Subjects. Eighteen subjects with normal or corrected-to-normal vision participated for course credit. All were right-handed with no lefthanded relatives in their immediate family, and all were naive about the purpose of the study.

Apparatus and Stimuli. Details of the apparatus and stimuli were the same as in Experiment 1, with the following modifications. The large global letter was constructed out of smaller letters (14-point Helvetica) arranged in a $5 \times 7$ matrix (subtending $2.1^{\circ} \times 3.0^{\circ}$ ), and stimuli were presented with their innermost edges at an eccentricity of $2.4^{\circ}$. These changes were introduced in an effort to make the local dimension more difficult to process and to establish global precedence. Stimuli were pre-
} 
sented at eight different locations: upper visual field, upper right visual field, right visual field, lower right visual field, lower visual field, lower left visual field, left visual field, and upper left visual field. All stimuli were presented equidistant from the fixation point.

Procedure. Details of the procedure were the same as in Experiment 1, with the following two exceptions. First, the letter E was designated as the target for all the subjects. Second, each block consisted of 160 trials: $32 \mathrm{~L}+\mathrm{S}+, 32 \mathrm{~L}+\mathrm{S}-, 32 \mathrm{~L}-\mathrm{S}+$, and $64 \mathrm{~L}-\mathrm{S}-$, again with an equal number of each trial type at each location. Thus, $60 \%$ of the trials contained a target. Each subject participated in three blocks of experimental trials, for a total of $\mathbf{4 8 0}$ trials.

\section{Results and Discussion}

The average error rate across conditions approached $5 \%$, so analyses were performed on both the RT and accuracy data. A two-way ANOVA was performed on the $\mathrm{RT}$ data, with stimulus type $(\mathrm{L}+\mathrm{S}+, \mathrm{L}+\mathrm{S}-, \mathrm{L}+\mathrm{S}-$, and $\mathrm{L}-\mathrm{S}-$ ) and stimulus location (upper, upper right, right, lower right, lower, lower left, left, and upper left visual fields) as within-subject variables. Significant main effects were obtained for stimulus type $[F(3,51)=36.42$, $p<.001]$ and stimulus location $[F(7,119)=13.29, p<$ $.001]$. The interaction between these two variables was also significant $[F(21,357)=1.86, p<.02]$. The main effect of stimulus type arose from the fact that RT was longer for $\mathrm{L}-\mathrm{S}+$ trials than for the other target trial types. The main effect of location arose from the fact that RT was longer for right, lower right, lower, and lower left visual field locations. Although simple RT is typically faster in the lower visual field (e.g., Payne, 1967), Schwartz and Kirsner (1982) reported an upper VFA for choice RT.

The nature of the interaction between stimulus type and location was addressed via a series of planned comparisons concentrating on the $\mathrm{L}+\mathrm{S}-$ and $\mathrm{L}-\mathrm{S}+$ stimulus types and on the comparison of left versus right and upper versus lower visual field performance. The RT and error data for these stimulus types are shown in Figures 2 and 3 . For the $\mathrm{L}+\mathrm{S}-$ stimulus types, requiring the processing of global information, there was no difference in RT between the upper and lower visual fields $(F<1)$. A comparison of the left and right visual fields yielded a

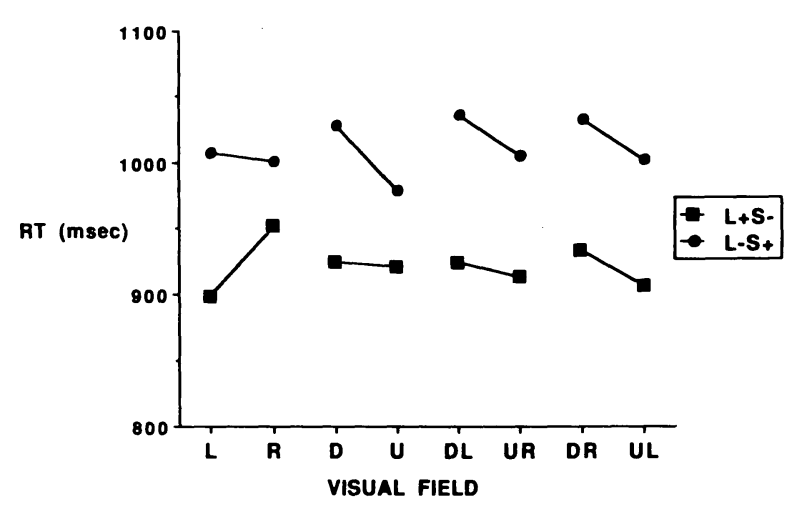

Figure 2. Reaction time as a function of stimulus type $(L+S-$ : global target only; $L-S+$ : local target only) and visual field location $(L=$ left, $R=$ right, $D=$ lower, $U=$ upper, $D L=$ lower left, UR = upper right, $D R=$ lower right, $U L=$ upper left) in Experiment 2.

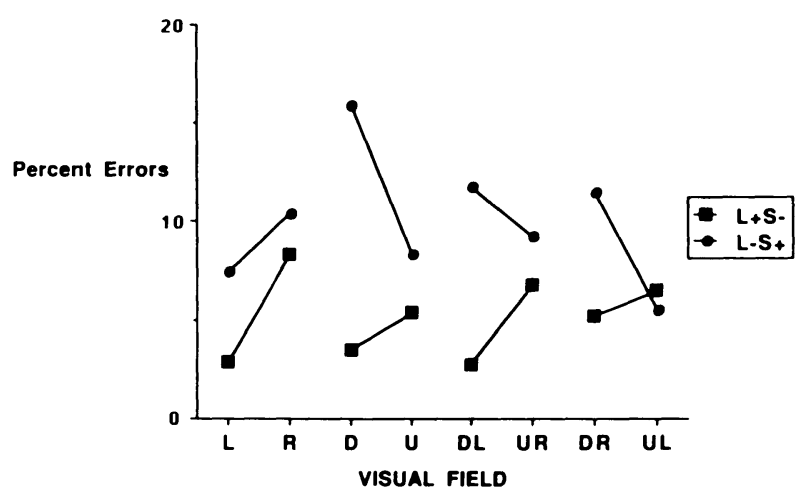

Figure 3. Percent errors as a function of stimulus type $(\mathrm{L}+\mathrm{S}-$ : global target only; $L-S+$ : local target only) and visual field location $(L=$ left, $R=$ right, $D=$ lower, $U=$ upper, $D L=$ lower left, $\mathrm{UR}=$ upper right, $\mathrm{DR}=$ lower right, $\mathrm{UL}=$ upper left) in Experiment 2.

significant left VFA $[F(1,17)=7.71, p<.02]$. A comparison of upper right versus lower left visual field performance yielded no difference $(F<1)$. For the $\mathrm{L}-\mathrm{S}+$ stimuli, requiring the processing of local information, a significant upper VFA was obtained $[F(1,17)=$ $6.25, p<.03$ ], but there was no left-right difference $(F<1)$. A comparison of upper right versus lower left visual field performance showed a marginally significant upper right VFA $[F(1,17)=3.35, p<.085]$. Thus, the predictions of the hypothesis were partially confirmed by the RT data. Although an upper VFA was obtained for local processing, there were no upper-lower differences in global processing. Similarly, although a left VFA was obtained for global processing, there were no left-right differences in local processing.

Similar analyses were carried out on the accuracy data. The overall ANOVA revealed a main effect of stimulus type $[F(3,51)=7.93, p<.001]$, no effect of location $[F(7,119)=1.60, p>.14]$, and a significant interaction between stimulus type and location $[F(21,357)=1.66$, $p<.04]$. This interaction arose from the presence of a left VFA for global processing $[F(1,17)=5.52, p<.04]$ and an upper VFA for local processing $[F(1,17)=5.54$, $p<.03]$. No other left-right, upper-lower, or upper right-lower left comparisons attained significance.

Due to the large number of conditions in this experiment, there were only 12 observations per trial type per location. This fact may have increased the variability of the data set and obscured certain effects. In an attempt to address this problem, post hoc analyses were performed for each trial type on data collapsed over locations sharing a common visual half-field. For example, data for locations in the upper right, right, and lower right visual fields were combined to provide a measure for overall right visual field performance based on 36 total observations. Although such analyses prevent examination of performance in particular oblique quadrants of the visual field, they do allow testing for the presence of overall upper versus lower and left versus right visual field differences, which served to test the reliability of the basic visual field 
effects obtained in Experiment 1. For $\mathrm{L}+\mathrm{S}-$ trials, a significant lower VFA was obtained for the accuracy measure $[F(1,17)=4.63, p<.05]$, as well as a significant left VFA for both the RT $[F(1,17)=7.20, p<.02]$ and accuracy $[F(1,17)=8.77, p<.01]$ measures. Finally, for $\mathrm{L}-\mathrm{S}+$ trials, a significant upper VFA was obtained for both the RT $[F(1,17)=11.90, p<.003]$ and accuracy $[F(1,17)=5.71, p<.03]$ measures, whereas there were no left-right differences for either measure.

\section{GENERAL DISCUSSION}

The results of the current experiments provide preliminary evidence for the existence of upper versus lower visual field advantages in the processing of local versus global visual information, and partially replicate previous findings of right versus left visual field differences. With regard to the upper-lower distinction, the results of Experiment 1 revealed that responses were faster to global information in the lower visual field and to local information in the upper visual field; in Experiment 2 , responses to global information were more accurate in the lower visua field, whereas responses to local information were faster and more accurate in the upper visual field. With regard to left-right visual field comparisons, Experiment 1 revealed a marginal trend toward a left VFA for global processing and no significant difference in local processing, whereas Experiment 2 yielded a left VFA for global processing in terms of both RT and accuracy; no visual field differences were obtained on either measure for local processing.

The lack of reliability of some of the effects (e.g., the presence of a lower VFA for global RT in Experiment 1 only; the lack of a significant right VFA for local processing in either experiment) is somewhat troublesome. One possibility is that, due to the relatively small number of trials per condition (especially in Experiment 2), the current experiments lacked sufficient power. Another possible reason for the lack of consistent left-right visual field differences is that such effects may be contingent upon the degree of location uncertainty. The typical laterality experiment employs only two stimulus locations, whereas the current experiments employed four and eight locations. Thus, the subjects were more uncertain on each trial as to which location would be stimulated, and this could have led to a reduction in the visual field difference (perhaps due to some unspecified attentional process). This is unlikely, however, because previous work has shown that positional uncertainty typically leads to larger, not smaller, effects (e.g., Davis, Kramer, \& Graham, 1983; Lamb \& Robertson, 1988).

In summary, partial support for the hypothesis of upper versus lower visual field differences in the processing of local-global visual information was obtained. Further testing needs to be done to determine the possible bases of these visual field differences. For example, recent work has demonstrated left-right visual field differences in the processing of both absolute and relative spatial frequency (Christman, Kitterle, \& Hellige, 1991; Kitterle, Christman, \& Hellige, 1990). These findings have been integrated with left-right visual field differences in local-global processing to suggest that part of the basis for local-global differences between the left and right visual fields may reside in differences in spatial frequency processing (e.g., Kitterle, Christman, \& Conesa, in press; Robertson \& Lamb, 1991).

This in turn suggests the feasibility of further investigation into the nature of upper-lower visual field differences in spatial frequency processing, as well as in other analogs of local-global processing. In fact, two recent papers have reported upper versus lower, as well as left versus right, visual field differences in spatial frequency processing. Edgar and
Smith (1990) reported differences in perceived spatial frequency as a function of visual hemifield. Of particular relevance to the current paper was the finding that the spatial frequency of stimuli presented in the lower and left visual fields was overestimated relative to stimuli presented in the upper and right visual fields. Similarly, Berardi and $\mathrm{Fi}$ orentini (1991) reported lower and left visual field superiorities in a spatial phase discrimination task. The stimuli they utilized were composed of relatively low spatial frequencies, which may have played a role in the fact that lower and left visual field superiorities were obtained. The exact relation of these results to the results of the current study is not clear, due to the different nature of the tasks employed. It is clear, however, that researchers studying left-right visual field differences in perceptual processing will need to address their relation to similar upper-lower visual field differences.

\section{REFERENCES}

Berardi, N., \& Fiorentini, A. (1991). Visual field asymmetries in pattern discrimination: A sign of asymmetry in cortical visual field representation? Vision Research, 31, 1831-1836.

BRYDEN, M. P., \& UNDERWOOD, G. (1990). Twisting the world by 90 degrees. Behavioral \& Brain Sciences, 13, 547-548.

Christman, S., Kitterle, F., \& Hellige, J. (1991). Hemispheric asymmetry in the processing of absolute versus relative spatial frequency. Brain \& Cognition, 16, 62-73.

Davis, E. T., Kramer, P., \& Graham, N. (1983). Uncertainty about spatial frequency, spatial position, or contrast of visual patterns. Perception \& Psychophysics, 33, 20-28.

EDGAR, G., \& SMITH, A. (1990). Hemifield differences in perceived spatial frequency. Perception, 19, 759-766.

Kitterle, F., Christman, S., \& Conesa, J. (in press). Hemispheric differences in the interference among components of compound gratings. Perception \& Psychophysics.

Kitterle, F., Christman, S., \& Hellige, J. (1990). Hemispheric differences are found in the identification, but not the detection, of low versus high spatial frequencies. Perception \& Psychophysics, 48, 297-306.

LAMB, M., \& RoberTson, L. (1988). The processing of hierarchical stimuli: Effects of retinal locus, locational uncertainty, and stimulus identity. Perception \& Psychophysics, 44, 172-181.

Navon, D. (1977). Forest before trees: The precedence of global features in visual perception. Cognitive Psychology, 9, 353-383.

PAYNE, W. (1967). Visual reaction times on a circle about the fovea. Science, 155, 481-482.

PrEvic, F. (1990). Functional specialization in the lower and upper visual fields in humans: Its ecological origins and neurophysiological implications. Behavioral \& Brain Sciences, 13, 519-575.

RoBerTSON, L., \& LAMB, M. (1991). Neuropsychological perspectives on part/whole organization. Cognitive Psychology, 23, 299-330.

SCHWARTZ, S., \& KIRSNER, K. (1982). Laterality effects in visual information processing: Hemispheric specialization or the orienting of attention? Quarterly Journal of Experimental Psychology, 34A, 61-77.

SERGENT, J. (1982). The cerebral balance of power: Confrontation or cooperation? Journal of Experimental Psychology: Human Perception \& Performance, 8, 253-272.

VAN KLEECK, M. H. (1989). Hemispheric differences in global versus local processing of hierarchical visual stimuli by normal subjects: New data and a meta-analysis of previous data. Neuropsychologia, 27, $1165-1178$ 